\title{
Brewster Anomalies: A Polarization-Induced Delocalization Effect
}

\author{
John E. Sipe, ${ }^{(a)}$ Ping Sheng, Benjamin S. White, and Morrel H. Cohen \\ Exxon Research and Engineering Company, Clinton Township, Annandale, New Jersey 08801 \\ (Received 3 November 1986)
}

\begin{abstract}
We predict that anomalously long localization lengths- which in some special cases diverge-can be expected for $p$-polarized light propagating through one-dimensional disordered structures under certain conditions. We give a physical argument for the existence of such anomalies, and support it by a longwavelength theory and Monte Carlo calculations.

PACS numbers: $42.20 .-\mathrm{y}, 03.40 . \mathrm{Kf}$
\end{abstract}

The demonstration that almost all stationary states in a one-dimensional disordered system are localized ${ }^{1}$ proceeds by the construction of a product of the transfer matrices of the system and application of Furstenberg's theorem ${ }^{2}$ on the product of random matrices. The proof of that theorem begins with the supposition that the matrices are irreducible; that is, in the simple case of twodimensional matrices, they cannot all be diagonalized by the same transformation.

It is easy to see why this assumption is ultimately crucial in establishing the localization of stationary states ${ }^{3}$ : If all the random transfer matrices were diagonal, the inhomogeneities in the system could not mix the state represented by one of the eigenvectors of those matrices with the state represented by the other. If those states corresponded to waves propagating to the right and left, the absence of scattering from one to the other would allow each to survive as a delocalized stationary state of the system. ${ }^{4}$

In this Letter we show that, under certain special circumstances, precisely this situation (or a situation close to it) can occur for the transport of light through disordered one-dimensional structures. The light is then no longer localized and the localization length diverges (or is at least enhanced orders of magnitude over its usual value). The effect is shown to have its origin in the inherent vector nature of the electromagnetic radiation and therefore is without analogy for the scalar waves.

To see physically how a beam of light might pass through a one-dimensional disordered structure without scattering, consider first the simple case of small inhomogeneities. For a dielectric constant $\epsilon(z)=\bar{\epsilon}+\hat{\epsilon}(z)$, with $|\hat{\epsilon}(z)| \ll \bar{\epsilon}$, we initially neglect $\hat{\epsilon}(z)$ and imagine a beam $i$ propagating at some angle $\theta$ with respect to the direction of inhomogeneity towards $+z$ [see Fig. 1(a)]; we consider light polarized in the plane of incidence as shown ( $p$-polarized light). Energy can be removed from $i$ by backscattering into a beam $s$; such a beam must make an angle $\theta$ from $-z$, since the system is translationally invariant in the $(x y)$ plane. An inhomogeneity $\hat{\epsilon}(z)$ that could lead to such scattering would do so by generating a polarization $\mathbf{P}(z)$ that, for $|\hat{\epsilon}| \ll \bar{\epsilon}$, would be given by $\mathbf{P}(z)=[\epsilon(z)-\bar{\epsilon}] \mathbf{E} / 4 \pi$, where $\mathbf{E}$ is the elec- tric field in beam $i$. If $\theta=45^{\circ}, \mathbf{P}(z)$ points in the direction that beam $s$ must propagate. Since dipoles cannot radiate in the direction they point, no backscattered wave can be generated.

If there is no dissipation in the system, the beam $i$ can then continue to propagate without extinction. The effect is closely analogous to the zero in reflectivity from a homogeneous medium at Brewster's angle, ${ }^{5}$ where the dipoles in the refracted beam point in the direction that the reflected beam would have to propagate, forbidding its generation. We shall see in the following that at $\theta=45^{\circ}$ in a random medium with $|\hat{\epsilon}| \ll \bar{\epsilon}$, as well as at a special angle in the particular case of a randomly layered binary medium with arbitrary $|\hat{\epsilon}|$, the localization length indeed diverges. More generally, the localization length is enhanced in the neighborhood of a certain angle

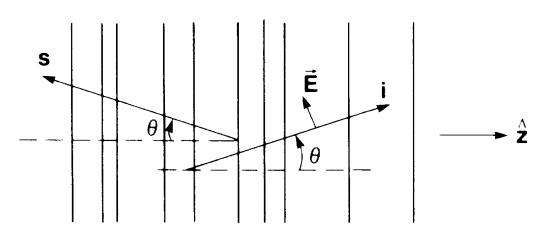

(a)

$\varepsilon(\mathbf{z})$
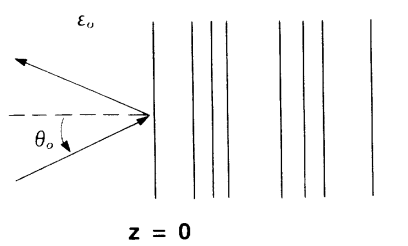

(b)

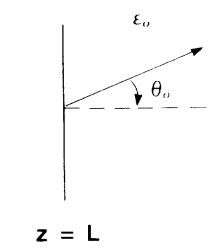

FIG. 1. (a) Schematic diagram indicating the incident beam $i$, the scattered beam $s$, and the polarization of the electric vector relative to the beams. The randomly layered medium has its normal in the $z$ direction. (b) Schematic picture defining the incident, the reflected, and the transmitted wave relative to the normal of a randomly layered medium with dielectric constant $\epsilon(z)$. 
$\theta_{A}$ by several orders of magnitude. From the above analogy, we refer to such an enhanced localization length as a "Brewster anomaly."

To treat the general problem, consider a medium with random dielectric constant $\epsilon(z)$ (real and positive but otherwise arbitrary) between $z=0$ and $z=L$, bounded by regions of uniform dielectric constant $\epsilon_{0}$ [see Fig. 1 (b)]. For a plane wave at frequency $\omega$ incident at $\theta_{0}$, a transfer-matrix formalism, which is a generalization of the familiar formalism at normal incidence, can be constructed. The state of the electromagnetic field is specified by a complex two-dimensional vector, the components of which refer to the amplitudes of the forwardand backward-propagating waves, respectively. The change in that state over a distance $d z$ is given by the product of $A(z) d z$ and the vector, where $A(z)$ is a $2 \times 2$ complex matrix. We write $A(z)=\bar{A}+\hat{A}(z)$, where $\bar{A}=\operatorname{diag}[i w,-i w]$ is the ensemble average of $A$ in diagonal form. Specializing now to $p$-polarized light $w$ $=\left(\tilde{\omega}^{2} \bar{\epsilon}-\bar{\epsilon} \bar{\epsilon}_{\text {inv }} k^{2}\right)^{1 / 2}, \tilde{\omega}=\omega / c, k$ is the wave-vector component in the $(x y)$ plane, and $\sin \theta_{0}=k / \tilde{\omega} \sqrt{\epsilon_{0}}$, and we have written $\epsilon(z)=\bar{\epsilon}+\hat{\epsilon}(z), \epsilon^{-1}(z)=\bar{\epsilon}_{\text {inv }}+\hat{\epsilon}_{\text {inv }}(z)$. Thus, $w$ is the wave-vector component perpendicular to the $(x y)$ plane in the effective medium approximation [neglect of $\hat{A}(z)$ ], and in this basis the vectors $(1,0)$ and $(0,1)$ represent waves directed to the right and left, respectively. Further, in the interaction picture defined by $\bar{A}$,

$$
\hat{A}_{I}(z)=i w\left(\begin{array}{cc}
m(z) & n(z) e^{-2 i w z} \\
-n(z) e^{2 i w z} & -m(z)
\end{array}\right),
$$

where

$$
\begin{aligned}
& m(z)=\frac{1}{2} w^{-2}\left[w^{2} \epsilon(z) / \bar{\epsilon}-k^{2} \bar{\epsilon} \hat{\epsilon}_{\mathrm{inv}}(z)\right], \\
& n(z)=-\frac{1}{2} w^{-2}\left[w^{2} \epsilon(z) / \bar{\epsilon}+k^{2} \bar{\epsilon} \hat{\epsilon}_{\mathrm{inv}}(z)\right] .
\end{aligned}
$$

The propagation of light through the disordered structure as described by the matrix of Eq. (1) is a random process which, for arbitrary magnitudes of $\hat{\epsilon}$ and $\hat{\epsilon}_{\text {inv }}$ but for variations in the inhomogeneities over distances small compared to the wavelength, converges in probability to a random walk in an abstract space. ${ }^{6}$ The diffusion coefficient for that random walk is related to the localization length $l$, yielding the result ${ }^{7,8}$

$$
l^{-1}=w^{2} \int_{0}^{\infty}\langle n(0) n(z)\rangle d z .
$$

One should note that in Eq. (3) the quadratic frequency dependence of $l^{-1}$ is attributable to Rayleigh scattering in $1 \mathrm{D}$. The magnitude scale of $l^{-1}$, on the other hand, is set by the integral of the correlation function for $n$. This low-frequency form of $l^{-1}$ is generally valid for classical wave transport in stratified random media. Obviously, the localization length increases if $n$ can be scaled down. Even more generally than Eq. (3), if $n(z)$ can be set equal to zero for all $z$, forward and backward waves do not mix [Eq. (1)], transfer matrices become diagonal, Furstenberg's theorem fails, and the localization length diverges as effective-medium theory becomes exact.

From Eqs. (2) and (3) we find

$$
\begin{array}{r}
l^{-1}=\frac{1}{4} \tilde{\omega}^{2} \bar{\epsilon}\left[c++\mu+c--(1-\mu)^{2} / \mu\right. \\
+2 c+-(1-\mu)],
\end{array}
$$

where $\mu \equiv 1-\bar{\epsilon}_{\text {inv }} \epsilon_{0} \sin ^{2} \theta_{0}$ and we have put

$$
c_{ \pm \pm}=\int_{0}^{\infty}\left\langle\hat{\eta}_{ \pm}(0) \hat{\eta}_{ \pm}(z)\right\rangle d z,
$$

with $\hat{\eta}_{+}(z) \equiv \hat{\epsilon}(z) / \bar{\epsilon}, \hat{\eta}_{-}(z)=\hat{\epsilon}_{\text {inv }}(z) / \bar{\epsilon}_{\text {inv }}$; we have assumed $c_{+-}=c_{-+}$. The maximum localization length occurs at $\theta_{0}=\theta_{A}$, where

$$
\bar{\epsilon}_{\text {inv }} \epsilon_{0} \sin ^{2} \theta_{A}=1-\left[c_{--}\left(c_{++}+c_{--}-2 c_{+-}\right)\right]^{1 / 2},
$$

and its value is given by

$$
\begin{array}{r}
2\left(\bar{\epsilon} \tilde{\omega}^{2} l_{\max }\right)^{-1}=\left[c_{--}\left(c_{++}+c_{--}-2 c_{+-}\right)\right. \\
\left.-\left(c_{--}-c_{+-}\right)\right]^{1 / 2} .
\end{array}
$$

For $l_{\max }$ to diverge, Eq. (7) requires

$$
c_{+-}^{2}=c++c--
$$

i.e., $\hat{\eta}_{+}$is a multiple of $\hat{\eta}_{-}$. Quite generally, this is the condition for it to be possible to set $n(z)$ equal to zero for all $z$ by the proper choice of $\theta_{0}$.

Such a divergence occurs, for example, in the limit of weak inhomogeneities where $\bar{\epsilon}_{\text {inv }} \simeq 1 / \bar{\epsilon}$ and $\hat{\epsilon}_{\text {inv }} \simeq-\hat{\epsilon} / \bar{\epsilon}^{2}$. In this limit Eq. (6) [or Eq. (2) directly] gives the result that $\theta_{0}=\theta_{A}$ at the divergence, where $\sin ^{2} \theta_{A}=\frac{1}{2} \bar{\epsilon} / \epsilon_{0}$; the beam indeed makes an angle of $45^{\circ}$ in the effective medium, in agreement with the earlier simple physical argument.

It is also possible to set $n(z)$ to zero for all $z$ by the proper choice of $\theta_{0}$ in the case of a binary randomly layered medium, with, however, arbitrary dielectric constants $\epsilon_{a}$ and $\epsilon_{b}$. Equation (6) [or Eq. (2) directly] requires that $\theta_{0}$ be set equal to $\theta_{A}$, where

$$
\epsilon_{0} \sin ^{2} \theta_{A}=\epsilon_{a} \epsilon_{b} /\left(\epsilon_{a}+\epsilon_{b}\right) .
$$

For such distributions, where it is possible to "zero $n(z)$ " by choice of angle, the reflectance of the structures can be found at that special angle since effectivemedium theory becomes exact. The Fresnel coefficients at the interfaces of the surrounding medium can then be extracted from the transformation that diagonalizes $\bar{A}$. We can obtain two amusing special cases by asking at what value $\epsilon_{0}$ should be set so that, for a binary mixture, at the special angle of the Brewster anomaly the reflectance vanishes-i.e., all the light is transmitted from one surrounding medium to the other. We find two solutions, $\epsilon_{0}=\epsilon_{a}$ or $\epsilon_{0}=\epsilon_{b}$. These results are physically obvious: If $\epsilon_{0}=\epsilon_{a}$, for example, the angle determined by 
Eq. (9) is just Brewster's angle for a ray impinging from medium $a$ to medium $b$. When the ray refracts into medium $b$, the angle at which it impinges on the next slab of material $a$ is precisely the Brewster's angle for that interface, and so the ray can pass completely through the structure by negotiating each interface without reflection.

For more general distributions, $\hat{\epsilon}^{-1}(z)$ is not simply proportional to $\hat{\epsilon}(z)$, and it is impossible to choose an angle of incidence so that $l_{\max }$ diverges. However, it is possible to get an $l_{\max }$ several orders of magnitude larger than $l$ at normal incidence. For example, in Fig. 2 we plot the localization length as a function of $\theta_{0}$ for a disordered structure consisting of slabs of random, uncorrelated thicknesses characterized by a probability density $P(d)=d_{0}^{-1} \exp \left(-d / d_{0}\right)$, with the ratio of the wavelength of light $\lambda$ in the surrounding medium $\epsilon_{0}$ to the mean spacing $d_{0}$ chosen to be $\lambda / d_{0}=48$. In a given

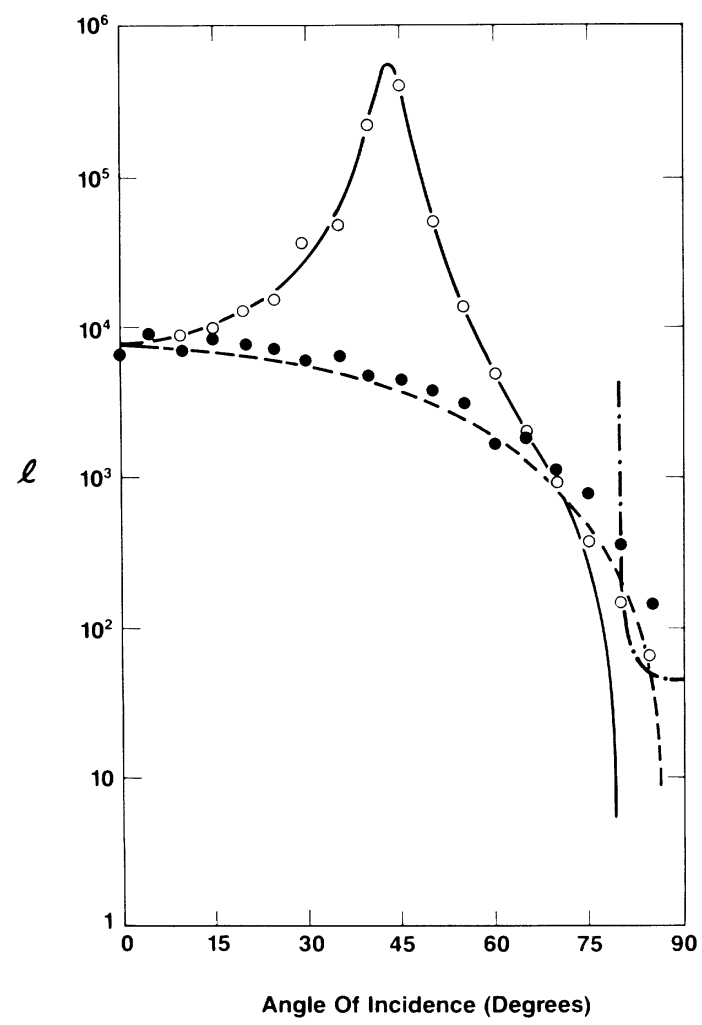

FIG. 2. Localization length in units of mean layer thickness $d_{0}$ plotted as a function of incidence angle for $\lambda=48 d_{0}$. The dielectric constant $\epsilon(z)$ of the random medium is defined in the text. Open circles are results of Monte Carlo simulation for $p$-polarized light, and solid circles are for $s$-polarized light. Solid and dashed lines are calculated from the long-wavelength theory for the $p$ - and the $s$-polarized waves, respectively. The dash-dotted curve indicates the evanescent decay length beyond the critical angle of the $p$-polarized light calculated in the effective-medium theory. slab the dielectric constant is taken as $\epsilon / \epsilon_{0}=(1+0.6 \xi)$, where $\xi$ is a random variable with a uniform distribution in $[-0.5,0.5]$. The solid line is the theoretical result [Eq. (4)], and the dots are the result of Monte Carlo calculations. They are in disagreement near the angle for total internal reflection in the effective-medium theory $(w=0)$ where $|n(z)|$ and $|m(z)|$ diverge and the theory ${ }^{6,7}$ leading to Eq. (3) breaks down. This interesting regime has been the subject of recent studies, ${ }^{9,10}$ and we shall return to it in a later communication. Away from this angle, Eq. (3) and the equations that follow from it should be valid, since $\lambda / d_{0} \gg 1$. Indeed, the theory correctly predicts a 2-order-of-magnitude enhancement at the Brewster anomaly with a fairly wide angular spread. In fact, this Brewster anomaly is a very robust phenomenon in the sense that we cannot find a distribution in $d$ and $\epsilon$ that would wash out the enhancement peak.

In Fig. 3 we plot the results for a binary randomly layered medium, the slab dielectric constants alternating between $\epsilon_{a}=\epsilon_{0}$ and $\epsilon_{b}=2.42 \epsilon_{0}$, with thicknesses again specified by the $P(d)$ above. Here we have chosen $\lambda / d_{0}=5$, and the theory of Eq. (3) (solid line) is only in qualitative agreement with the Monte Carlo calculations

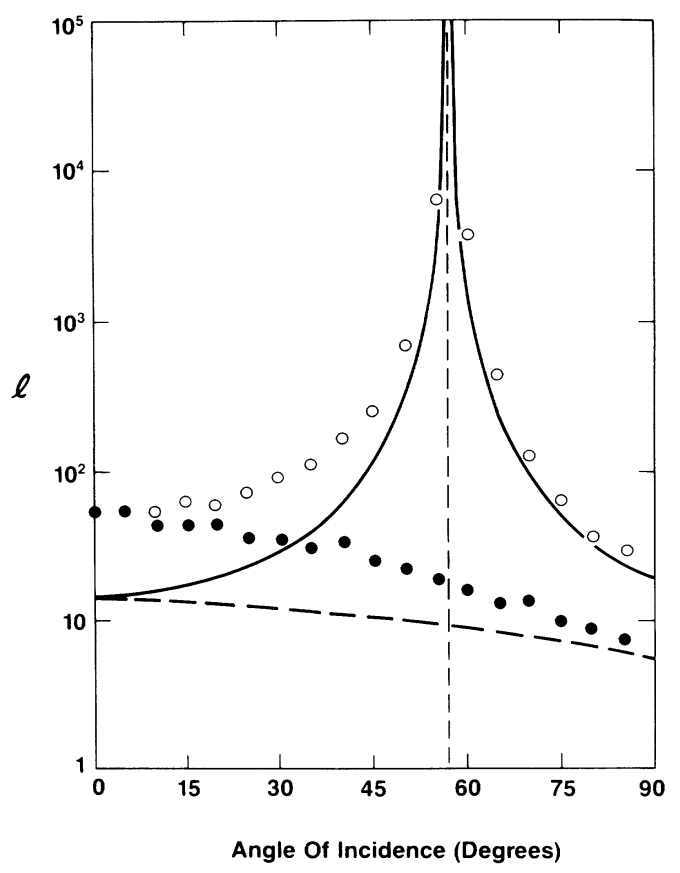

FIG. 3. Localization length in units of mean layer thickness $d_{0}$ plotted as a function of incidence angle for wavelength $\lambda=5 d_{0}$. The random medium is a binary layered system with $\epsilon_{a}=\epsilon_{0}$ and $\epsilon_{b}=2.42 \epsilon_{0}$. Open circles are results of Monte Carlo simulation for $p$-polarized light, and solid circles are for $s$ polarized light. Solid and dashed lines are calculated from the long-wavelength theory for the $p$ - and the $s$-polarized waves, respectively. 
(dots). Nonetheless, the divergence in $l$ is clearly indicated. It should be remarked here that while the analytical formula used in this paper is based on the lowfrequency approximation, the predicted divergence in $l$ for a random binary system is nevertheless valid for all frequencies as can be reasoned from its physical basis elucidated earlier. For general distributions of $\epsilon$ and $d$, the angular variation of $l$ for $\lambda \ll d_{0}$ can be calculated by the high-frequency formula as presented in Ref. 8, Eq. (11), with a mapping of the variables. ${ }^{11}$

Note finally that the results for light polarized perpendicular to the plane of incidence ( $s$ polarized) show no such anomaly (see Figs. 2 and 3). None, of course, would be expected from the physical argument given at the start of this Letter. [The $\hat{A}(z)$ for $s$-polarized light at arbitrary angle of incidence is given by Eq. (1), but with $w=\left(\tilde{\omega}^{2} \bar{\epsilon}-k^{2}\right)^{1 / 2}$ and $m(z)=n(z)$ $=\frac{1}{2} \tilde{\omega}^{2} \hat{\epsilon} / w^{2}$.]

In conclusion, we have shown that significant delocalization of $p$-polarized light in one-dimensional disordered structures can be expected near certain angles where scattering between forward and backward propagating rays is inhibited. At and near such "Brewster anomalies," the localization length can be enhanced over its value at normal incidence by orders of magnitude and, in certain special cases, can even diverge. Monte Carlo calculations are in good agreement with a long-wavelength theory, and it would be very interesting to see an experimental confirmation of this effect.

(a) Permanent address: Department of Physics and Erindale College, University of Toronto, Toronto, Ontario, Canada M5S 1A7.

${ }^{1}$ H. Matsuda and K. Ishii, Prog. Theor. Phys. Suppl. 45, 56 (1970)

${ }^{2}$ H. Furstenberg, Trans. Am. Math. Soc. 108, 377 (1963).

${ }^{3}$ J. M. Ziman, Models of Disorder (Cambridge Univ. Press, Cambridge, 1979), Chap. 8.

${ }^{4}$ G. Theodorou and M. H. Cohen, Phys. Rev. B 13, 4597 (1976).

${ }^{5}$ M. Born and E. Wolf, Principles of Optics (Pergamon, New York, 1964).

${ }^{6}$ R. Burridge, G. Papanicolaou, and B. White, SIAM J. Appl. Math. (to be published).

${ }^{7}$ R. Burridge, G. Papanicolaou, and B. White, in Proceedings of the University of Minnesota Workshop on Random Media, 1985 (to be published).

${ }^{8}$ P. Sheng, B. White, Z. Q. Zhang, and G. Papanicolaou, Phys. Rev. B 34, 4757 (1986).

${ }^{9}$ W. E. Kohler, SIAM J. Appl. Math. 46, 464 (1986).

${ }^{10}$ J. P. Bouchaud and P. LeDoussal, J. Phys. A 19, 797 (1986).

${ }^{11}$ Density $\rho \rightarrow \epsilon / c$, and compressibility $K^{-1} \rightarrow 1-\epsilon^{-1} \epsilon_{0}$ $\times \sin ^{2} \theta_{0}$. 